\title{
Effective Virtual Teaching through the Interactive and Inexpensive Teaching Laboratory Data Management (TLDM) System
}

\author{
Derek Yau Chung Choy, Gaurav Subedi, Daniel G. McClement, Dhaneshwarie Kannangara \\ The University of British Columbia \\ dhanesh.kannangara@ubc.ca
}

\begin{abstract}
The COVID-19 pandemic has shifted a significant number of on-campus and in-person activities to an online, virtual setting. This has caused difficulty in achieving the same learning outcomes in the absence of in-person interaction, particularly for lab courses. In 2014, the Chemical and Biological Engineering department at The University of British Columbia developed and implemented a Teaching Laboratory Data Management (TLDM) system to improve the delivery and effectiveness of lab-based courses. The TLDM system guides students through experimental calculations and automates a significant amount of grading for instructors. The TLDM system aided the department to better adapt to online lab courses and was integral to the virtual instruction of lab courses during the COVID-19 pandemic. Through conducting surveys and group feedback sessions with students, teaching assistants (TAs) and instructors, we found that the TLDM system has been well received by all three groups of stakeholders for use in online lab courses, and provides several key benefits. Namely, it helps students understand the calculations involved in experiments and provides an effective substitute for in-person lab activities, while reducing workload for TAs and instructors. The TLDM system can potentially be a great tool to complement online courses around the world in an inexpensive way.
\end{abstract}

Keywords: Online laboratory courses, data management, economical, interactive, Microsoft Excel ${ }^{T M}$

\section{INTRODUCTION}

The outbreak of severe acute respiratory syndrome coronavirus 2 (SARS-CoV-2), also named coronavirus disease 2019 (COVID-19), in late 2019 severely disrupted lives across the globe and initiated a paradigm shift in social interactions. Many universities restricted in-person classes and shifted towards online delivery. As a part of this response, many higher educational institutions adopted the use of video-based conferencing platforms, (such as Zoom Cloud Meetings, Cisco Webex, Microsoft Teams) and learning management systems (LMS) (such as Canvas, Blackboard, Learn and Google Classroom) as part of their online learning environments [1][2][3]. The University of
British Columbia embraced the changing digital landscape and adopted the use of Canvas Collaborate Ultra and Zoom Cloud Meetings to conduct many of its classes online during this strategic shift. However, there were still many hurdles to overcome. The Teaching Laboratory Data Management (TLDM) system [4], developed in 2014 prior to the COVID-19 outbreak, had already been a part of the successful delivery of laboratory courses at the Chemical and Biological Engineering Department (CHBE) during in-person learning, and has played an even more prominent role for online learning.

In this paper, we outline our experience with using the TLDM system for the online delivery of laboratory courses, and highlight some of the benefits of using the system. We also discuss current challenges and future opportunities for the application of this system to online education, based on data and comments collected through surveys and feedback sessions with students, TAs, and instructors.

\section{THE TLDM SYSTEM}

The TLDM system is a tool designed to help with the challenges of data management associated with the collection and post-processing of the large amount of raw data from laboratory courses [4]. This system has served the CHBE department well after its adoption in 2014 and was extensively used in second and third-year in-person laboratory courses. Raw data generated during subsequent years was archived and used to help with the continuous improvement of laboratory teaching and the identification of problems with equipment.

The main goal of the TLDM system is to serve as a unified platform for students, TAs and instructors to work with laboratory data. The core components of the system are located within several Microsoft Excel ${ }^{\mathrm{TM}}$ files (four for each experiment) that are designed to help students record their unique raw data generated in the laboratory, and complete the numerical calculations required to derive the final results expected for each experiment. This system guides students through calculations without instructor support as the provided sample raw data and expected calculated values enable students to obtain instant feedback on the accuracy of their calculations. It also provides instructors and TAs 
with grading assistance by generating answer keys specific to each student's unique raw data, improving grading efficiency and accuracy while significantly decreasing time spent. Optional scaffolding features allow the system to be tailored to students with varying levels of ability.

The TLDM system was moved to the forefront of the program's lab instruction when courses had to be quickly moved online during the COVID-19 pandemic. Time that students would normally spend performing experiments was designated instead to filling out the TLDM files. Raw data archived by the TLDM system over past years was provided to the students enrolled in the online-only version of the courses, and was used for calculations in lieu of the data that would have normally been generated during in-person experiments.

\section{CHALLENGES}

Although many classroom-based courses can be easily offered online through learning management systems, there are many hurdles to overcome for delivering laboratory courses virtually. Laboratory courses are an important aspect of engineering degree programs as they provide indispensable practical exposure for students and complement concepts learnt in the classroom [5]-[9].

Undergraduate laboratory courses in the CHBE department allow students to translate theoretical knowledge into practice. Students are encouraged to cultivate problem solving skills through group laboratory exercises, and effective communication skills through drafting individual and group reports. The development of technical laboratory skills is one of the primary challenges associated with online laboratory learning, as it is difficult to truly replace the experience gained in a physical lab environment. In addition, virtual teaching brings forth the question of active student engagement that must be addressed during online delivery $[10,11]$.

Committed to continuing to deliver these experiences online, the CHBE department initiated a strategic shift of its laboratory courses from in-person delivery towards digital-based data analysis in 2020. The transition was sudden (within an academic year) and hence challenging for both students and teachers, especially for those who are experiencing online teaching and learning for the first time. In this new environment, students are forced to "double down" on the development of certain skills and behaviours (adaptability, computer literacy, remote learning, and self-motivation) as they become more important than ever. Instructors are also challenged to design and deliver a new learning environment where teaching, classroom discussions and evaluation of outcomes are now all conducted virtually. Instructors have no opportunity to meet with students face-to-face to establish rapport, and additional time is needed for new preparation activities (such as drafting and updating teaching notes specific for virtual teaching) in order to continue to convey key concepts in the new learning environment.

\section{SOLUTIONS}

One goal of teaching virtual lab courses is to mimic in-person instruction where students gain handson and transferable skills while working closely with instructors. To teach in this new environment, instructors must engage students in a timely and frequent manner, providing feedback and creating a collaborative learning atmosphere. Teaching of hands-on knowledge now relies on tools such as digital media and online laboratory simulations, while web-based quizzes and calculation templates also help students to prepare for online laboratory classes. When equipped with interactive content, these tools help students self-correct and selflearn, reducing the time TAs and instructors need to commit for office hours. Additionally, a potential constraint for instructors is limited budgetary resources to invest in new digital tools that facilitate virtual teaching and support student learning. Therefore, there is a preference for inexpensive tools that are available and accessible for everyone.

In-line with this strategic shift, the Teaching Laboratory Data Management (TLDM) system became one of the most important tools for the online delivery of CHBE laboratory courses (CHBE 263, 264, 362, 364, 365 and 366) during the fall 2020 and winter 2021 semesters, operating within the online learning environment based on Canvas [4, 12]. The system was initially developed in 2014 to improve the delivery of CHBE lab courses for students and teachers: it addressed the need for 1) a greater focus on data management, 2) enhanced teaching on the instructor's side and 3) a means for collaborative exchange and learning for students. Serendipitously, the TLDM system was found to be extremely effective at enabling and enhancing virtual laboratory learning.

Providing students with a way to check their calculations using sample data makes the TLDM system engaging for students, and provides them an opportunity to self-correct and self-learn. When students work in groups to fill out their TLDM files using Microsoft Excel $^{\mathrm{TM}}$ or Google Sheets ${ }^{\mathrm{TM}}$, it sparks discussion about concepts behind the experiment. In this way, the TLDM system helps mimic the self-directed nature of in-person labs, in stark contrast to most other online courses where students spend the significant amount of time receiving unidirectional information when they listen to lectures.

The self-guided nature of the TLDM system also reduces the number of instructor and TA hours required for teaching as students can work more independently. Additionally, instructors have access to a modified TLDM file which automatically grades the students' calculations, eliminating grading time spent on 
reproducing and checking tedious calculations, which provides teachers more time to give students feedback on written lab reports and other forms of assessment. The TLDM system's ability to reduce teaching and grading hours, coupled with its minimal upfront cost (can be implemented solely using Microsoft Excel $\left.{ }^{\mathrm{TM}}\right)$, makes it a great tool that is easy and practical to implement.

\section{METHODOLOGY}

Data was collected quantitatively through surveys, and qualitatively through group feedback sessions as well as one-on-one interviews with three focus groups: instructors, TAs and undergraduate students. The survey results collected from the secondyear CHBE students experiencing online learning and the TLDM system for the first time formed the primary basis of the results and discussion for the current paper. $\mathrm{CHBE}$ second-year students complete two laboratory courses (one per semester). The survey was conducted anonymously with second-year students $(n=124)$ who used the TLDM system in the second semester at the time of the survey. These students had not previously taken any in-person CHBE lab courses. Responses from a similar survey, conducted with a CHBE second-year inperson lab course, were used for comparison. The inperson second-year survey results presented in a previous paper introducing the TLDM system [4] are used for this comparison study. Similarly, a separate survey was conducted for TAs and instructors across several CHBE courses that use the TLDM system. This survey had a total of $n=14$ responses. Survey questions were selected to investigate the effectiveness of the TLDM system in meeting its primary objectives: 1) helping students perform and understand data analysis involved in their labs, 2) enhancing student's online learning experience, 3) facilitating online discussion between students and teachers, and among students, and 4) helping teaching teams reduce grading time and errors. The survey results are presented in the next section.

Qualitative data was also collected from students, TAs, and instructors. Online meetings with groups of TAs $(n=4)$, instructors $(n=4)$ and undergraduate students $(n=8)$ were conducted separately with openended discussions on the transition to online teaching/learning and the effectiveness of the TLDM system. The eight third-year students selected for the student focus group discussion had prior experience with using the TLDM system during in-person laboratory learning (2019-2020), as well as experience with using the system for virtual learning during the COVID-19 pandemic (2020-2021). Participants were informed of the discussion topic (use of the TLDM system for online laboratory courses) in advance to encourage well- thought-out responses. The online focus groups' responses were collated and are presented in the next section.

\subsection{CHBE second-year laboratory}

\subsection{Course content before COVID-19}

Due in part to the early innovation and adoption of learning management systems at UBC [13], the CHBE department was quick in implementing tools for blended learning, which consists of face-to-face teaching combined with various online elements. $\mathrm{CHBE}$ offered blended learning for many of its courses, including laboratory courses. The CHBE second-year laboratory courses were transitioned to blended learning in 2002 with the WebCT LMS. Blended learning offers several advantages and they have been adopted worldwide [14]-[16].

The laboratory courses in the CHBE secondyear curriculum are designed to introduce laboratory practises for students in both chemical and biological streams while laboratory courses in the third-year introduce students to concepts and techniques used in environmental engineering, biotechnology and process engineering. Problem solving and communication skills are key learning outcomes in all CHBE lab courses. Prior to COVID-19, most laboratory courses consisted of a host of lab activities/workshops, and experiments planned around lab safety, experimental design, data collection, analysis and observation. Each second-year laboratory experiment before COVID-19 consisted of in-person prelab, wet lab and post-lab sessions (Table 1). The pre-lab sessions consisted of a thorough review of the concepts and layout of the experiments provided by the TA. The labs were taught with experimental write-ups that include rich content, such as images and short video clips of the apparatus and experimental setup. Students were expected to read manuals and review the video clips that were prepared to help with the understanding of key experimental concepts. The pre-lab sessions were also followed by group pre-lab assignments that had to be submitted before the wet lab sessions. The wet lab required students to be present for 2 (Term 1) to 4 (Term 2) hours in the laboratory, where they completed the experiments in groups of 3 to 4 students. Upon the students' arrival at the laboratory, each group had to draw a flowchart of the procedure of the assigned experiment solely from memory on a whiteboard, and explain it to the TA before they could turn on any equipment. The TA assigned grades for each student's lab preparedness based on the quality of their pre-lab 
Table 1: Second-year lab sessions before COVID-19 and the pivot to online learning

\begin{tabular}{|l|c|c|c|}
\hline & Outcomes/Deliverables & Before COVID-19 & During COVID-19 \\
\hline Pre-lab Quizzes & Preparation for Experiment & N/A & Online Individual \\
\hline Pre-lab & Introduction to Experiment & In-person TA Presentation (In Lab) & Online TA Presentation (Synchronous) \\
\hline $\begin{array}{l}\text { Pre-lab } \\
\text { Assignment }\end{array}$ & Group Assignment & In-person Group Work & Online Group Work (Asynchronous) \\
\hline $\begin{array}{l}\text { Calculation } \\
\text { Quizzes }\end{array}$ & Preparation for Calculations & N/A & Online Individual \\
\hline $\begin{array}{l}\text { Wet Lab } \\
\text { Raw Data Collection }\end{array}$ & $\begin{array}{l}\text { In-person Group Work (Face-to-face) } \\
\text { (In Lab) }\end{array}$ & $\begin{array}{c}\text { Online Group Work with TLDM } \\
\text { Completed TLDM Files }\end{array}$ \\
\hline $\begin{array}{l}\text { TLDM Files } \\
\text { Submission }\end{array}$ & Report with Appendices & In-person or Online Group Work & Online Group Work \\
\hline Post-lab & Final Report & In-person Group Work in Classroom & $\begin{array}{c}\text { Online Group Work (Synchronous) } \\
\text { (In Breakout Rooms) }\end{array}$ \\
\hline Report Submission & Online (Asynchronous) & Online (Asynchronous) \\
\hline
\end{tabular}

assignments and their ability to answer questions at the beginning of the labs.

The students were given access to the TLDM files online before each experiment, which helped them understand the raw data collected during wet lab sessions. Students were recommended to review the empty raw data and final results tables, calculation templates and sample answers from the TLDM files [4]. This prepared them well for the wet lab session. In the following week, students met in the post-lab session to write reports, which involved performing calculations based on their experimental data. The TLDM system was an important tool used by students in their effort to understand key concepts and expectations before the experiments, as well as after the experiments when they were completing calculations with their raw data.

\subsection{Course content during COVID-19 (pivoting to online format)}

The sudden need for the delivery of all laboratory courses online was challenging for the CHBE department, and the early adoption of the Canvas LMS and the TLDM system prior to COVID-19 was fortuitous in hindsight. Since many of the components of the laboratory courses were already offered in the blended system before COVID-19 (Table 1), strategies were formulated to identify a replacement for the collection of raw data during wet lab sessions. Most sessions were held synchronously and minimal deviation from procedures used during inperson laboratory education was allowed in order to allocate equal time and effort towards the course. Synchronous delivery of the wet lab sessions was achieved by tapping into the past raw data repositories established by the TLDM system. This data was provided to students who work collaboratively in a group to complete the calculations with the aid of the TLDM system.

Online quizzes substituted the TAs' evaluation of students' preparedness before experiments, whereas students were previously queried in-person by the TAs prior to starting the wet lab sessions. Students completed online quizzes administered through the Canvas course site for individual preparedness before attending pre-lab and wet lab sessions. The pre-lab and wet lab sessions consist of TA presentations followed by a meeting in breakout rooms to complete group pre-lab assignments, calculations and reports. Video recordings were made available to students who were unable to be present due to unavoidable circumstances.

\section{RESULTS AND DISCUSSION}

\subsection{Reported Success}

According to the survey and collated responses from focus groups, the transition to online laboratory teaching was generally successful. Together with videos and presentations, the TLDM system greatly enhanced students' engagement and immensely helped them through tedious calculations while learning virtually. Overall, students shared a positive outlook with online learning and the use of the TLDM system. Most students $(80 \%$, Figure 1) who used the TLDM system for the first time online found it immensely useful for their calculations. The TLDM system greatly aided experimental understanding by providing a self-explanatory framework for conducting difficult and tedious calculations. With limited teaching 
hours provided during online teaching, providing step-bystep explanations for calculations to multiple groups would have been a very taxing job without the TLDM system. Thus, it was not surprising that a large majority of students (62\%, Figure 1) found the TLDM system to be helpful during online learning. Students strongly voiced the utility of the TLDM system in saving their time during online delivery (73\%, Figure 1). Most students (80\%, Figure 1) expressed the need to further extend the implementation of the TLDM system beyond CHBE labs to non-laboratory courses involving engineering calculations.

A high majority of second-year students $(67 \%$, Figure 1) were inexperienced in Excel when they started the course. This highlighted a need to develop spreadsheet skills in the curriculum. The TLDM system helps to develop the necessary skillset in the second-year by providing structured spreadsheets. However, the scaffoldings are removed subsequently in the third and fourth-years.

Figure 2 compares the survey responses of second-year CHBE students during in-person and online delivery. The in-person survey response is from 2014 at a time when the TLDM system was newly introduced for inperson CHBE teaching labs and has been referenced herein [4]. The TLDM system was useful for students in both inperson and online delivery (88\% and $80 \%$, Figure 2). Almost all students recommended the TLDM system for other lab courses (96\% and 80\%, Figure 2).

An overwhelming majority of the teaching team $(92 \%$, Figure 3$)$ found the TLDM system useful for teaching lab courses, and all who participated would recommend this system to another instructor. A large number of them $(85 \%$, Figure 3$)$ found it useful at reducing time spent in grading reports. One objective of the TLDM system is to facilitate discussion between students and teachers by having a shared spreadsheet. Instructors and students can clarify points of confusion surrounding calculations by pin-pointing the exact cells of interest in the Excel $^{\mathrm{TM}}$ spreadsheets of the TLDM system files. A majority (62\%, Figure 3) agreed on the TLDM system's usefulness in this regard and this was a topic revisited by the focus groups.

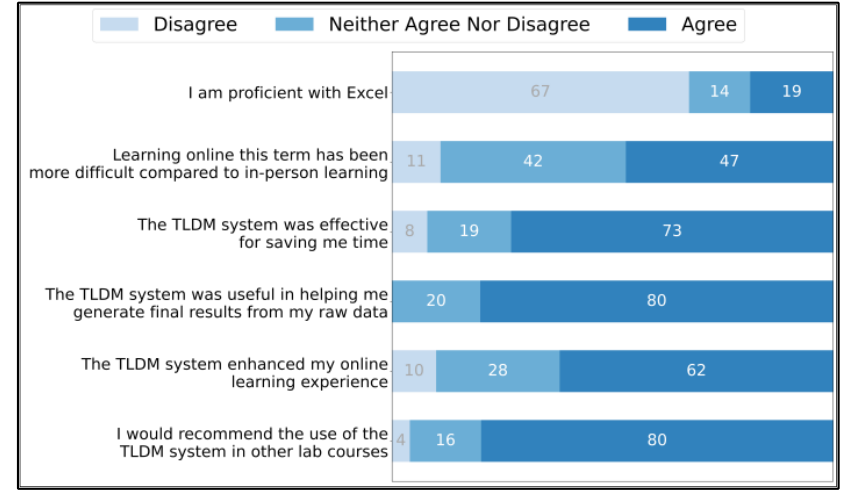

Figure 1. Survey responses from second-year students who had used the TLDM system for their online lab course ( $n=112,90 \%$ response rate), expressed as percentages

The focus groups provided significant insight on the transition to online learning and the use of the TLDM system therein by student and teaching teams. The single most important insight from the students was in the area of student engagement and enhanced learning. Students found that dedicated time for utilizing the TLDM system with TAs and instructors provided them with a better understanding of key concepts related to data analysis. This was exemplified by the focus group with third-year students who commented on using the TLDM system retrospectively during in-person learning and compared against using it during online learning. They reported that the task of performing the experimental data analysis during in-person lab sessions was delegated to certain group members while others would focus on writing the lab reports. The students said this specialization resulted in many group members not having a chance to learn from performing the data analysis themselves, while others had a heavy workload from solely completing all the calculations. Students felt that this was less of a problem in an online setting because of how the TLDM system was being used. Student time which normally would have been spent performing experiments in the lab was converted to dedicated time periods where lab groups would meet to work on the TLDM files together. This structure led to every group member playing an active role in performing calculations, and led to more group discussions around the calculations, which students reported helped their understanding. These designated work periods also allowed students to consult TAs and course instructors if they had any questions about their calculations. In the past, the TLDM calculation file would be filled out on students' own time which resulted in less group discussion and less access to TAs and instructors for assistance. 


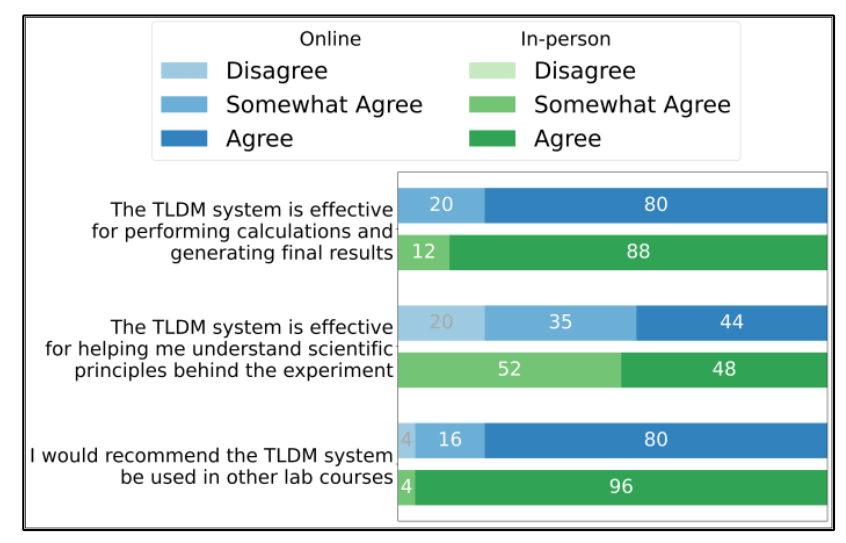

Figure 2. Survey responses from second-year students who had used the TLDM system for an online lab course $(n=112)$ vs. students who had used the TLDM system in the same course in-person $(n=25)$, expressed as percentages

Students in the focus group were asked whether it was more difficult to use the TLDM system for online experiments when they had not personally collected the data, and hence might be less familiar with the physical meaning of certain measurements. Responses indicated that students found the structure of the TLDM system helpful for understanding the data. Having every raw data measurement neatly organized in a single file (D file) and having every value they need to derive listed in the answer file (A file) helped students understand why each measurement was needed and how it related to the values they needed to calculate.

Survey responses also included comments. One second-year student who completed the survey (online learning) left a comment which captured the sentiment of most responses: "The TLDM system is fantastic, actually. Data analysis is rapid and relatively straightforward, and any specific issues can be pinpointed and diagnosed very quickly. There is a lot of setup required for the TLDM file, but, everything considered, it is an excellent tool."

The TA focus group also shared important insights on the advantage of using the TLDM system during online teaching. TAs particularly mentioned how the focus on data analysis yielded better communication with students that strongly helped them with the overall understanding of the experiment. Overall, the TAs shared that dedicated time to fill the TLDM files with students improved the effectiveness of the TLDM system greatly, when compared against in-person learning when this component was not mandatory.

The course instructor focus group offered a great amount of insight into areas of improvement and potential new applications of the TLDM system. Instructors shared ways to improve the effectiveness of online learning and the TLDM system. One instructor pointed out the necessity to train teaching teams on the use of the TLDM system files, and when possible, dedicated hours should be allocated for the maintenance and continuous improvement of these files. Indeed, revisiting the TLDM system files may be necessary when instructors may want to change components of the experiment. Instructors were specifically asked whether they thought students had a harder time using the TLDM system when they had not personally collected the data and may have a harder time understanding the physical meaning behind certain measurements or calculated values. They reported that they did not observe students having any difficulty with understanding the data.

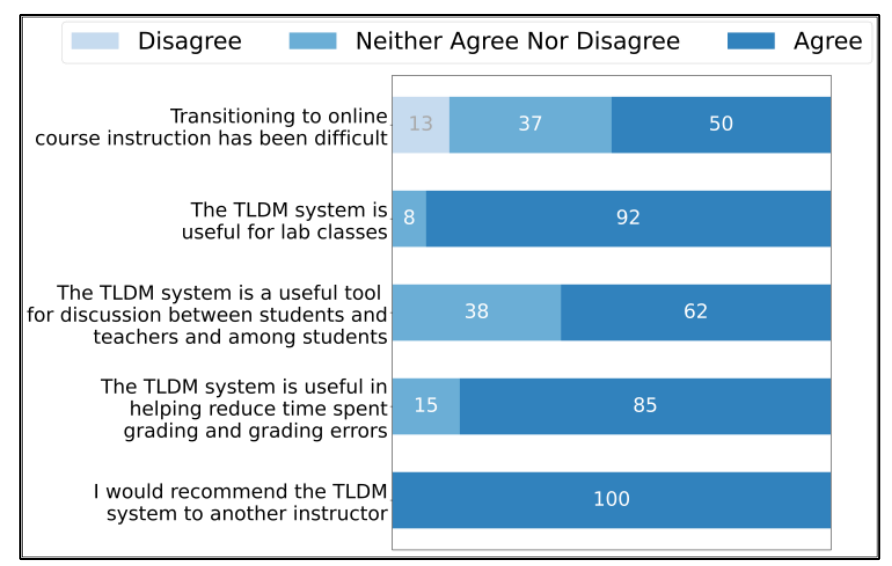

Figure 3. Survey responses from TAs and course instructors $(n=15)$, expressed as percentages

\subsection{Reported Challenges}

Given the enormous challenge in providing a substitute for hands-on laboratory experience for a large student population, transitioning to online classes presented a colossal problem. Although the use of learning management systems made many aspects of the abrupt transition to online learning easier, continuous learning and adaptation to newer pedagogical approaches may be necessary to keep offering quality learning and engagement for students. Active student engagement during online teaching is particularly challenging. In this regard, the TLDM system was chosen to enhance and retain active student engagement during online learning. Many challenges that occurred in this transition were noted through the survey and focus group discussions. The transition to online classes was difficult and confusing to many students $(47 \%$, Figure 1$)$. There were also differences in the expertise level of Excel ${ }^{\mathrm{TM}}$ users, with 19\% (Figure 1) of students reporting limited experience with Excel $^{\mathrm{TM}}$. Both these factors can compound to make online CHBE laboratory courses (which generally involve large amounts of data analysis) particularly challenging for 
students. Students in both online and in-person classes reported some effectiveness of the TLDM system (44\% vs $48 \%$ ) in helping them understand scientific principles behind experiments (Figure 2). A small portion of students $(20 \%)$ who used the TLDM system online (compared to none in the in-person classes) reported it to be not useful at all for understanding scientific principles behind experiments. One possible explanation for this difference between the in-person and online cohorts is the different relationship students have with the TLDM system. Inperson, students perform the experiment themselves and generate their own raw data. The TLDM system is merely seen by them as a tool for data analysis. In contrast, students who take the course virtually may see the TLDM file as being a more central component of the course, since it is the primary environment where they spend the majority of their time in the course. Students who learn virtually may generally have a harder time understanding the scientific principles behind the experiment without performing experiments themselves, and inadvertently associate this shortcoming with the TLDM system, expecting it to do more in helping them understand the scientific principles. This suggests that no matter how much we try to improve the effectiveness of virtual learning, it can never truly replace in-person teaching.

Half of the teaching team surveyed $(50 \%$, Figure

3 ) found the transition to online course instruction difficult. One instructor noted that students struggled more with error analysis calculations since courses moved online. We think students may have less intuition performing error analysis calculations when they do not have the opportunity to perform the experiments themselves to get an understanding of where errors are introduced during experiments. We propose a solution to this problem through the introduction of sensitivity analyses in the TLDM system in Section 6.4.

Another instructor noted how they found errors made their way into the TLDM system answer keys after being passed around multiple different TAs and instructors over the years. Instructors found the large spreadsheets difficult to debug and troubleshoot as there are often many cells that need to be examined. To solve this problem, we recommend implementing version control for the TLDM system files. A master copy of the TLDM system files should be kept somewhere safe in case any changes are accidentally made to the version in use. If any updates are warranted on the TLDM system files (such as due to changes in an experimental procedure), the changes made should be documented for version control and made on the secured master TLDM system files.

\subsection{Other considerations}

The COVID-19 pandemic has emphasized the importance of online learning and many educational institutions are accepting the transition to this mode of teaching. Although many pedagogical techniques may have to be adopted for effective online teaching, some considerations have to be taken during its development and implementation. Chief among these is the socio-economic factor. The educational sector has been hard-hit with the pandemic, and schools with lower financial capabilities will struggle to provide an effective learning environment to its students. The TLDM system is based solely on Microsoft Excel ${ }^{\mathrm{TM}}$ and does not require any other proprietary software components, which means that it can be widely used by schools as well as students at minimal cost. As the online mode of teaching may persist for the foreseeable future, having a repository of data becomes more important than ever; the TLDM system is an excellent tool for archiving both the raw data produced from experiments as well as calculated results submitted by students.

\subsection{Future opportunities}

Discussions with students, TAs, and instructors have given us ideas on how the TLDM system can be further improved or extended to new applications. As previously mentioned, some instructors wished that the TLDM system could be used to help students develop greater awareness of the uncertainties associated with calculated values. Once the TLDM calculation template file is completed for a certain set of calculations, it can be used to generate calculated results based on any set of raw data. Therefore, the system can potentially be used to illustrate the impact of uncertainties in measured values (which are part of the input raw data) on final calculated results. The TLDM system can also serve as a "sandbox" for students to freely explore what-if scenarios and conduct sensitivity/risk analyses on experiments.

From the repository of past raw data, instructors can find sets of "good" or "bad" data, as well as data sets that are associated with certain unique scenarios (such as specific errors made during the experiment or issues with equipment used). Students can input these different sets of raw data into their completed calculation template files, and instructors can use the TLDM system as a tool to highlight the relationship between experimental steps/issues and the calculated results rather than just using the TLDM system as a tool for performing one-time calculations.

The idea of using the TLDM system as a sandbox to play around with how changes to raw input data affect calculated values can be further extended beyond laboratory courses. Non-laboratory courses that involve iterative or tedious numerical calculations could also benefit from the use of the TLDM system, shifting the focus from pure number crunching to setting up teaching that promotes the fundamental understanding of concepts. The TLDM system could allow for more interesting homework assignments that involve teachers asking students to test and comment on relationships between critical variables in situations where it would have 
normally been impractical for large amounts of repetitive tedious calculations to be done manually.

During the instructor focus group discussions, one instructor mentioned that they found the TLDM system excellent for well-defined experiments but wondered how it would perform for more open-ended laboratory courses, such as those where students could design their own experiments and procedures. Although the TLDM system was indeed initially designed for well-defined labs where all students execute identical experimental procedures and collect the same measurements, some instructors in the department are already experimenting with more openended uses of the TLDM system. For example, one secondyear lab involves the synthesis of biodiesel and the analysis of the synthesized samples' physical properties. Students are encouraged to bring in a reagent of their choice (such as coconut oil, avocado oil, etc.) to perform the experiment with. This flexibility gives students the opportunity to create and test their own hypotheses, such as "the biodiesel produced using avocado oil will have a higher viscosity than biodiesel produced using vegetable oil because...”. The TLDM system approach could be translated to experiments where students create their own procedures and choose which variables they would like to manipulate. As long as the types of data collected by each student are consistent (such as viscometer readings, the temperature inside a reactor vessel, etc.), the TLDM system can still be used effectively.

\section{CONCLUSIONS}

The TLDM system is a useful tool for helping students work with and understand laboratory experimental data, even when they have not had the opportunity to generate and collect the raw data themselves due to the suspension of in-person learning. The TLDM system continues to serve as a great platform for facilitating group discussions whether they take place inperson or online, and provides structure during virtual lab periods that students would normally have used to conduct in-person experiments. The TLDM system has already been used in six different CHBE second-year and thirdyear teaching laboratory courses during the COVID-19 pandemic. Some of the lab classes had over 125 students who joined the online sessions from across Canada as well as from other countries in the world. Overall, the TLDM system serendipitously played a critical role in the delivery of CHBE laboratory courses in a completely virtual format during the COVID-19 pandemic, with little to no additional investment of financial and human resources, while receiving overwhelmingly positive feedback from students, TAs and instructors. The system demonstrates strong potentials for continued use for either in-person or virtual teaching/learning, as well as for both laboratory as well as non-laboratory engineering courses that involve complex numerical calculations.

\section{ACKNOWLEDGEMENTS}

We gratefully acknowledge the financial support of the University of British Columbia Teaching and Learning Enhancement Fund (TLEF).

We would also like to extend our sincere gratitude to the students, TAs, and instructors who took the time out of their busy schedules to attend our focus groups and provide their feedback on the TLDM system. 


\section{REFERENCES}

[1] E. V. Jones, C. G. Shepler, and M. J. Evans, "Synchronous Online-Delivery: A Novel Approach to Online Lab Instruction," J. Chem. Educ., 2021.

[2] W. Flynn, N. Kumar, R. Donovan, M. Jones, and P. Vickerton, "Delivering online alternatives to the anatomy laboratory: Early experience during the COVID-19 pandemic," Clin. Anat., 2021.

[3] J. W. J. Ang, "Scaffolded Inverse Blended Learning: An Approach to Teach an Online General Chemistry Course," J. Chem. Educ., 2020.

[4] D. Y. C. Choy, J. Sibley, and D. Kannangara, "The Teaching Laboratory Data Management (TLDM) System," Proc. Can. Eng. Educ. Assoc., pp. 1-8, 2019.

[5] D. Pusca, R. J. Bowers, and D. O. Northwood, "Handson experiences in engineering classes: The need, the implementation and the results," World Trans. Eng. Technol. Educ., 2017.

[6] L. D. Feisel and A. J. Rosa, "The role of the laboratory in undergraduate engineering education," in Journal of Engineering Education, 2005.

[7] J. Ma and J. V. Nickerson, "Hands-on, simulated, and remote laboratories," ACM Comput. Surv., 2006.

[8] S. A. Clemons, "Developing online courses for visual/kinesthetic learners: A case study," Instr. Technol. Distance Learn., vol. 1, no. 11, pp. 51-62, 2004.

[9] J. R. Brinson, "Learning outcome achievement in non- traditional (virtual and remote) versus traditional (handson) laboratories: A review of the empirical research," Comput. Educ., 2015.

[10] A. Khan, O. Egbue, B. Palkie, and J. Madden, "Active learning: Engaging students to maximize learning in an online course," Electron. J. e-Learning, 2017.

[11] K. A. Smith, S. D. Sheppard, D. W. Johnson, and R. T. Johnson, "Pedagogies of engagement: Classroom-based practices," in Journal of Engineering Education, 2005.

[12] D. Kannangara and J. Sibley, "Transforming undergraduate chemical engineering laboratories," Proc. Can. Eng. Educ. Assoc., pp. 1-8, 2019.

[13] D. PETERS, "Learning management systems are more important than ever," University Affairs, 2021.[Online].Available:

https://www.universityaffairs.ca/features/featurearticle/learning-management-systems-are-moreimportant-than-ever/. [Accessed: 09-Mar-2021].

[14] C. Dziuban, C. R. Graham, P. D. Moskal, A. Norberg, and N. Sicilia, "Blended learning: the new normal and emerging technologies," Int. J. Educ. Technol. High. Educ., 2018.

[15] W. Zhang and C. Zhu, "Review on Blended Learning: Identifying the Key Themes and Categories," Int. J. Inf. Educ. Technol., 2017.

[16] C. R. Graham, "Blended learning systems: Definition, current trends, and future directions," in Handbook of blended learning: Global perspectives, local designs, 2006. 